\title{
Characterization of the p3-VvAGL11 Marker for Stenospermocarpic Seedlessness in Euvitis $\times$ Muscadinia Grape Hybrid Progenies
}

\author{
Patrick J. Conner ${ }^{1}$ and Gunawati Gunawan \\ Department of Horticulture, University of Georgia-Tifton Campus, 4604 Research Way, Tifton, GA \\ 31793 \\ John R. Clark \\ Department of Horticulture, University of Arkansas, 316 Plant Sciences Building, Fayetteville, AR \\ 72701
}

\begin{abstract}
ADDITIONAl INDEX wORDs. Vitis rotundifolia, Muscadinia rotundifolia, molecular breeding, simple sequence repeat
Abstract. Stenospermocarpic seedlessness from Vitis vinifera $\mathbf{L}$. is being introgressed into muscadine grape (Vitis rotundifolia Michx.) germplasm through the use of a cross-fertile hybrid of the two species. Recently, a sequencetagged site (STS) molecular marker, p3_VvAGL11, has been developed which enables detection of the dominant allele controlling stenospermocarpic seedlessness in $V$. vinifera. This marker was evaluated in six Euvitis Planch. $\times$ Muscadinia Planch. hybrid progenies to determine its association with seedlessness in this material. The presence of the 214-bp seedlessness-associated p3_VvAGL11 allele in seedling vines resulted in a nearly 3-fold reduction in mean seed fresh weight (MSFW) and significantly reduced mean seed weight per berry (MSWB), percent berry weight composed of seed (BWCS), and mean berry weight (MBW). When the lack of lignified seed was used as the determinant of seedlessness, the p3_VvAGL11 marker was able to correctly judge seedlessness in $\approx 85 \%$ of the progeny. Analysis of seedlessness in the progenies was hampered by poor vigor and fruiting ability of the hybrid seedlings. The p3_VvAGL11 marker shows potential to speed the introduction of the stenospermocarpic seedlessness into Muscadinia germplasm by identifying seedless progeny at the seedling stage.
\end{abstract}

The genus Vitis L. contains two subgenera, Euvitis (bunch grapes) and Muscadinia (muscadine grapes). Muscadinia consists of just three species: $V$. rotundifolia, the common muscadine grape known throughout the southeastern United States, Vitis munsoniana (Simpson ex Munson) M.O. Moore, a semitropical variant of $V$. rotundifolia native to southern Florida, and Vitis popenoei J.H. Fennel a tropical native to southern Mexico. The muscadine grape is the only commonly cultivated member of the Muscadinia subgenus. Muscadine is an important native North American grape species planted mainly in the southeastern United States. The muscadine grape differs from the familiar bunch grape (Vitis labrusca L. and $V$. vinifera) by the presence of smaller clusters, unbranched tendrils, and berries with thick skins and a unique fruity aroma (Basiouny and Himelrick, 2001). In addition, the Muscadinia species have 40 chromosomes $(2 x=2 n=40)$, whereas Euvitis have $38(2 x=2 n=38)$.

Seedlessness is a key trait in the development of freshmarket Euvitis cultivars. There are two types of seedlessness that can occur in grapes. In parthenocarpic seedlessness, the fruit develops without fertilization, whereas in stenospermocarpic seedlessness, the seeds abort a few weeks after fertilization (Ledbetter and Ramming, 1989). Parthenocarpic grapes lack seed traces, whereas a range of sizes of seed and seed traces

Received for publication 7 Feb. 2018. Accepted for publication 6 Apr. 2018. We thank Paige Catotti for technical help in obtaining the data used for the preparation of this manuscript and David Ramming and the U.S. Department of Agriculture table grape breeding program for the donation of pollen from advanced seedless selections.

${ }^{1}$ Corresponding author. E-mail: pconner@uga.edu. is found in crosses between stenospermocarpic seedless and seeded cultivars, as well as in crosses between two stenospermocarpic cultivars (Striem et al., 1996). In these progenies, classification of seeded vs. seedless individuals is influenced by factors such as texture of the berry flesh, degree of development of the seed, and sclerification of integuments (Ledbetter and Ramming, 1989). Seed trace size can also be influenced by vine maturity, rootstock, and environmental conditions (Ledbetter and Ramming, 1989).

Currently, most seedless cultivars of Euvitis are derived from the cultivar Sultanina (also known as Thompson Seedless), which is stenospermocarpic (Karaagac et al., 2012). Genetic control of seedlessness is a matter of some contention, but the most widely accepted theory is that seedlessness is conditioned by the presence of a dominant allele at a single locus named SDI for "seed development inhibitor," inhibiting the development of the seed by regulating three recessive genes (Bouquet and Danglot, 1996). Quantitative trait loci studies have confirmed that seedlessness is primarily regulated by the SDI locus on chromosome 18 , which is responsible for $50 \%$ to $70 \%$ of total phenotypic variance for the trait. New seedless cultivars can be obtained by crossing a seeded parent to a seedless self-fertile pollen parent. These crosses typically produce from $10 \%$ to $40 \%$ seedless progeny, depending on the definition of seedlessness used by the breeder. To improve the percentage of seedless progeny and reduce the size of seed traces, many breeding programs have begun crossing two seedless cultivars; progeny are then obtained through embryo rescue and tissue culture. However, this recurrent selection reduces the genepool and can lead to inbreeding depression, and so seeded $\times$ seedless crosses are still sometimes used (Karaagac et al., 2012). 
The development of seedless fresh-market muscadine cultivars has been a long-term goal of muscadine breeding programs (Lane, 1997; Lu et al., 1993). Breeding seedless muscadines, however, has been hampered by the lack of a source of stenospermocarpic seedlessness in the species. The only seedless member of Muscadinia is 'Fry Seedless', which is both parthenocarpic and male sterile, and therefore cannot be used as a parent in breeding (Basiouny and Himelrick, 2001).

There is a long and rich history in the use of Euvitis $\times$ Muscadinia hybrids, primarily to improve the disease resistance of $V$. vinifera with genes from $V$. rotundifolia. There has also been interest in improving the fruit quality of $V$. rotundifolia with genes from $V$. vinifera (Dunstan, 1963). Traits of interest include more stable fruit pigments (Goldy et al., 1986), improved fruit quality (Dunstan, 1963), and stenospermocarpic seedlessness (Lane, 1997; Lu et al., 1993). The introduction of stenospermocarpic seedlessness from Euvitis into Muscadinia is hampered by the difference in chromosome numbers between Euvitis and Muscadinia. Euvitis $\times$ Muscadinia hybrids frequently have stunted vines, and fruit-set tends to be very low and often parthenocarpic (Dunstan, 1963; Patel and Olmo, 1955). However, earlier researchers have noted that there is considerable variation in the fertility of individual hybrid vines indicating greater and lesser degrees of compatibility in certain combinations and a total lack of it in others (Dunstan, 1963; Fry, 1964; Jelenković and Olmo, 1968). NC74CO49-10 is a female vine developed from a cross between the $V$. rotundifolia cultivar Magnolia and a Euvitis $\times$ Muscadinia hybrid (Goldy et al., 1986). By crossing NC74CO49-10 to stenospermocarpic seedless $V$. vinifera cultivars and then backcrossing these hybrids to muscadine, stenospermocarpic seedlessness has been introduced into muscadine germplasm (Bloodworth, 2014).

Previous research has located a functional candidate gene, $V v A G L 11$, for stenospermocarpic seedlessness in $V$. vinifera (Mejía et al., 2011) which was subsequently renamed VviAGL11 (Grimplet et al., 2014). VviAGL11 corresponds to a D-lineage MADS-box gene whose ortholog in Arabidopsis thaliana L. was shown to be involved in the control of ovule identity (Rounsley et al., 1995). VviAGL11 expression peaks at 2 and 4 weeks after fruit-set in the dual endotesta layer of the seeds in seeded grapevines, whereas reduced expression was suggested as the cause of stenospermocarpic seedlessness in 'Sultanina' and derived cultivars (Malabarba et al., 2017; Mejía et al., 2011). An STS marker, p3_VvAGL11, was designed to perform genetic analysis of INDELs in the regulatory region of VviAGL11. It marks a (GAGA) motif in the regulatory region of VviAGL11 and has a high correlation with seedlessness. Subsequent tests on $V$. vinifera germplasm by Bergamini et al. (2013) to validate the p3_VvAGL11 marker found only eight of 475 genotypes that were seeded but carried the dominant p3_VvAGL11 allele associated with seedlessness. In addition, each seedless cultivar that was tested did carry the seedlessness-associated allele. The lack of false negatives and very low rate of false positives indicate that this marker is suitably effective for performing early negative selection of stenospermocarpy.

Extensive crossing work with NC74CO49-10 has been undertaken at the University of Georgia to introduce seedlessness from multiple Euvitis sources into muscadine breeding germplasm. In addition to seedlessness, it is desired to introgress genes for new flavors, bunch architecture, improved texture, and more stable pigments. As was found in previous work, Euvitis $\times$ Muscadinia hybrid seedlings vary greatly in vigor and fertility. In addition, analysis of seedlessness has been hampered by the presence of parthenocarpic berries on many vines, making it difficult to differentiate between parthenocarpic berries on sterile vines and seedless stenospermocarpic berries. The objective of this study is to determine if the p3_VvAGL11 marker developed for detection of the dominant allele of the SDI locus can be used in Euvitis $\times$ Muscadinia hybrid seedlings to predict the presence of stenospermocarpic seedlings.

\section{Materials and Methods}

Plant material. Vines of the germplasm collection and crossing experiments were grown at the University of GeorgiaTifton Campus located in Tifton, GA (lat. 33 53' 7.69" N, long. $\left.83^{\circ} 25^{\prime} 20.30^{\prime \prime} \mathrm{W}\right)$. The parents and population sizes of the crosses Ga. 12-11, Ga. 12-13, Ga. 13-11, Ga. 14-17, Ga. 14-18, and Ga. 14-20 are shown in Table 1. Several sources of stenospermocarpic seedlessness were used in this study. Vitis vinifera parents Y139-139-04, Y127-200-05, and C49-96 are advanced $V$. vinifera table grape selections obtained from Dr. David Ramming and the U.S. Department of Agriculture Table Grape Breeding Program. 'Jupiter' was released by the University of Arkansas as an eastern U.S.-adapted seedless table grape and has some $V$. labrusca in its pedigree (Clark and Moore, 1999). 'Concord Seedless' is an eastern U.S. adapted seedless table grape, which is unrelated to 'Concord' (Reisch et al., 1993), but likely has V. labrusca in its pedigree. A-3006 is an advanced seedless selection from the University of Arkansas grape breeding program with a complex pedigree that is believed to include both Euvitis and Muscadinia. Pollen of $V$. vinifera seedless selections Y139-139-04, Y127-200-05, and C49-96 were obtained from Dr. Ramming. Pollen of the seedless selection A-3006 was obtained from the University of Arkansas. Pollen from 'Jupiter' and 'Concord Seedless' were obtained from vines growing in Tifton, GA. The crosses were made in 2012-14 with the year the cross was made indicated by the first number in the cross number.

Phenotypic Characterization. Phenotypic characterization of seedling progenies was undertaken in 2015-17. However, because of low vigor of the hybrid seedlings, most seedlings only fruited in one or two of the 3 years and many seedlings were too weak to produce fruit at all. Quantitative analysis was performed on berry and seed development traits. A maximum of 20 berries [berry number $(\mathrm{BN})$ ] were randomly sampled from each fruitful vine. If less than 20 berries were produced, the minimum number that were sampled and the vine's data that were included in the study was five. If the vine produced a mixture of small seedless berries and larger berries with seeds, only the larger seeded berries were sampled because of the smaller berries being seedless likely due to parthenocarpy. The berry sample was weighed [total berry weight (TBW)]. All seeds and seed traces were removed from the berry sample and counted [seed number (SN)] and weighed [total seed fresh weight (TSFW)]. From these measurements, $\mathrm{MSFW}=\mathrm{TSFW} / \mathrm{SN}, \mathrm{MBW}=\mathrm{TBW} / \mathrm{BN}, \mathrm{MSWB}=\mathrm{TSFW} / \mathrm{BN}$, and $\mathrm{BWCS}=100 \times \mathrm{TSFW} / \mathrm{TBW}$ were calculated.

Qualitative analysis of the seed was also performed. Each seed or seed trace was segregated into one of four categories: 1) not evaluable aborted seeds or ovules, 2) rudimentary seeds, 
Table 1. Segregation ratios for the p3-VvAGL11 marker for stenospermocarpic seedlessness, progeny numbers, and fruit production in six Euvitis $\times$ Muscadinia grape hybrid progenies.

\begin{tabular}{|c|c|c|c|c|c|c|c|}
\hline Progeny & $\begin{array}{c}\text { Female parent } \\
\text { (p3_VvAGL11 genotype) }\end{array}$ & $\begin{array}{c}\text { Male parent } \\
\text { (p3_VvAGL11 genotype) }\end{array}$ & $\begin{array}{l}\text { Progeny } \\
\text { (no.) }\end{array}$ & $\begin{array}{l}\text { Vines with } \\
\text { fruit (no.) }{ }^{\mathrm{z}}\end{array}$ & $\begin{array}{l}\text { Progeny } \\
\text { genotypes }\end{array}$ & $\begin{array}{c}\text { Seedling } \\
\text { (no.) }\end{array}$ & $\begin{array}{c}\chi^{2 \mathrm{y}} \\
(P \text { value }) \\
\end{array}$ \\
\hline \multirow[t]{4}{*}{ Ga. $12-11$} & NC74CO49-10 & Y139-139-04 & 99 & 11 & $194 / 204$ & 21 & 0.76 \\
\hline & $194 / 212$ & $204 / 214$ & & & $204 / 212$ & 26 & $(0.86)$ \\
\hline & & & & & $212 / 214$ & 21 & \\
\hline & & & & & Offtype ${ }^{x}$ & 9 & \\
\hline \multirow[t]{4}{*}{ Ga. $12-13$} & NC74CO49-10 & Y127-200-05 & 191 & 43 & $194 / 204$ & 43 & 3.02 \\
\hline & & & & & $194 / 214$ & 46 & \\
\hline & & & & & $212 / 214$ & 42 & \\
\hline & & & & & Offtype ${ }^{x}$ & 3 & \\
\hline \multirow[t]{3}{*}{ Ga. $13-11$} & NC74CO49-10 & C49-96 & 59 & 16 & $194 / 204$ & 12 & 4.14 \\
\hline & $194 / 212$ & $204 / 214$ & & & $194 / 214$ & 19 & $(0.25)$ \\
\hline & & & & & $212 / 204$ & 9 & \\
\hline & & & & & $212 /$ null & 16 & \\
\hline & & & & & $212 / 214$ & 6 & \\
\hline & & & & & Offtype $^{\mathrm{x}}$ & 1 & \\
\hline \multirow[t]{5}{*}{ Ga. $14-18$} & NC74CO49-10 & Concord seedless & 50 & 6 & 194/null & 12 & 0.23 \\
\hline & $194 / 212$ & null/214 & & & $194 / 214$ & 11 & $(0.97)$ \\
\hline & & & & & $212 /$ null & 11 & \\
\hline & & & & & $212 / 214$ & 13 & \\
\hline & & & & & Offtype $e^{x}$ & 3 & \\
\hline \multirow[t]{3}{*}{ Ga. $14-20$} & NC74CO49-10 & A-3006 & 126 & 11 & $194 / 204$ & 22 & 16.6 \\
\hline & $194 / 212$ & $204 / 214$ & & & $194 / 214$ & 22 & $(<0.001)$ \\
\hline & & & & & $212 / 204$ & 49 & \\
\hline
\end{tabular}

${ }^{\mathrm{z}}$ Number of vines that produced $>5$ berries in any year 2015-17 and were sampled for phenotypic data.

${ }^{\mathrm{y}}$ Chi square of a $1: 1: 1: 1$ segregation ratio and its associated probability value.

${ }^{\mathrm{x}}$ Genotype has alleles not found in either parent.

3) complete but unlignified seed, and 4) complete and lignified seeds (Bergamini et al., 2013). The average for all seed for the vine is reported as mean seed classification (MSC).

Molecular characterization. DNA extraction of leaf tissue was performed as described previously (Conner et al., 2017). Genetic analysis of the STS marker p3_VvAGL11 was performed as described by Bergamini et al. (2013), except that amplification was performed in a thermal cycler (Eppendorf Scientific, Westbury, NY) programmed for 35 amplification cycles $\left(94^{\circ} \mathrm{C}, 30 \mathrm{~s} ; 57^{\circ} \mathrm{C}, 1 \mathrm{~min} ; 72^{\circ} \mathrm{C}, 1 \mathrm{~min}\right)$, followed by an 8 -min extension cycle at $72^{\circ} \mathrm{C}$, with maximum ramping speed between temperatures, and the M13 forward primer was labeled with either HEX or FAM fluorophore. Amplicons were mixed with 500 ROX Size Standard (Applied Biosystems, Foster City, $\mathrm{CA}$ ), denatured, and sized at the Georgia Genomics Facility using the Fragment Analyzer ${ }^{\mathrm{TM}}$ Automated CE System (Advanced Analytical, Ankeny, IA). Subsequently, Geneious (version 8.04; Biomatters, Newark, NJ) was used for allele size estimation by manual scoring of individual peaks.

Data analysis. Segregation ratios of the p3_VvAGL11 marker in the Muscadinia $\times$ Euvitis hybrid progenies were tested for goodness of fit to expected ratios by chi square tests. Offtype vines, those containing allele sizes not found in either parent, were removed from all further analysis. Because relatively few seedlings fruited in each population (Table 1), phenotypic measurements from the six hybrid progenies were grouped together for analysis. Normality of the phenotypic traits was determined using the Shapiro-Wilk test, and equal variance was tested with the Brown-Forsythe equal variance test. The effect of year on phenotypic traits was determined by the Mann-Whitney rank sum test using vines that produced berries in 2015 and 2016 and vines that produced berries in 2016 and 2017. There were too few vines that produced berries in 2015 and 2017 to compare those years. Because there were no significant year effects, phenotypic data from all 3 years were combined into a single data set. When vines fruited in more than a single year, all data points were combined for that individual. The effect of the 214-bp seedlessness-associated p3_VvAGL11 allele on the phenotypic trait with a normal distribution (MBW) was determined by a $t$ test. The effect of the 214-bp seedlessness-associated p3_VvAGL11 allele on phenotypic traits with nonnormal distributions (MSFW, MSWB, BWCS, and MSC) was determined by the Mann-Whitney rank sum test. Associations between phenotypic traits were determined using Spearman's rank-order correlation of the mean values. 


\section{Results and Discussion}

Primers for the p3_VvAGL11 marker were tested on a group of 38 Euvitis clones from our germplasm collections consisting of 20 seeded and 18 seedless selections and cultivars (Supplemental Table 1). All of the seedless Euvitis accessions displayed a 214-bp allele, whereas none of the seeded Euvitis accessions had this allele (Supplemental Table 1). This result indicates that the 214-bp allele corresponds to the 198-bp seedlessness-associated allele of Mejía et al. (2011) and the 216-bp allele (198-bp plus 18-bp M13 primer tail) of Bergamini et al. (2013). It is not clear why the seedlessness-associated allele is 214 bp rather than the 216 bp produced by Bergamini et al. (2013) when the same primers were used, but the difference may be the result of differences in sizing platforms or dye chemistry (Sutton et al., 2011) used in this study vs. those used by Mejía et al. (2011) and Bergamini et al. (2013). The original allele sizes are presented in parenthesis in Table 2 but will hereafter be reported with the sizes produced in this study. Evaluation of the p3_VvAGL11 marker on the Euvitis collection revealed eight different alleles (Table 2). Seven of these alleles with sizes of 192, 194, 200, 204, 208, 212, and 214 bp have been reported previously (Bergamini et al., 2013). In addition to these, we detected a null allele in three different seedless Euvitis cultivars (Supplemental Table 1). In each of these cultivars, a single peak corresponding to $214 \mathrm{bp}$ was produced. The presence of the null allele was demonstrated by a $1: 1: 1: 1$ segregation pattern in the progenies that had these cultivars as a parent (Table 1). These results agree with those of Bergamini et al. (2013) in that the 214 allele was only found in a heterozygous condition, despite crossing among seedless cultivars and subsequent embryo rescue being a common breeding method. The p3_VvAGL11 marker was tested in 99 Muscadinia cultivars and selections (Supplemental Table 1), and bands of expected size were amplified in all samples without primer modification. Only three alleles were produced in the Muscadinia germplasm, with sizes of 194, 196, and 200 bp. Interestingly, the 196-bp allele, which is by far the most common in Muscadinia (Table 2), has not been detected in Euvitis, and the 194- and 200-bp alleles are also relatively uncommon in Euvitis (Table 2, Bergamini et al., 2013). Importantly, the 214-bp seedlessness-associated allele was not detected in any Muscadinia germplasm; thus the 214-bp

Table 2. Allele frequencies of the p3_VvAGL11 sequence-tagged site marker for stenospermocarpic seedlessness in Euvitis and Muscadinia grape germplasm.

\begin{tabular}{|c|c|c|c|c|c|c|}
\hline \multirow{2}{*}{$\begin{array}{l}\text { Allele } \\
\text { size }^{z}\end{array}$} & \multicolumn{2}{|c|}{ Muscadinia } & \multicolumn{2}{|c|}{ Seeded Euvitis } & \multicolumn{2}{|c|}{ Seedless Euvitis } \\
\hline & Count & Frequency & Count & Frequency & Count & Frequency \\
\hline $192(176)$ & 0 & 0 & 5 & 0.11 & 0 & 0 \\
\hline $194(178)$ & 29 & 0.15 & 6 & 0.15 & 1 & 0.03 \\
\hline $196(180)$ & 153 & 0.77 & 0 & 0 & 0 & 0 \\
\hline $200(184)$ & 16 & 0.08 & 1 & 0.02 & 2 & 0.06 \\
\hline 204 (188) & 0 & 0 & 21 & 0.46 & 9 & 0.25 \\
\hline 208 (192) & 0 & 0 & 4 & 0.09 & 2 & 0.06 \\
\hline 212 (196) & 0 & 0 & 3 & 0.07 & 1 & 0.03 \\
\hline 214 (198) & 0 & 0 & 0 & 0 & 18 & 0.50 \\
\hline Null & 0 & 0 & 0 & 0 & 3 & 0.08 \\
\hline Total & 198 & & 40 & & 36 & \\
\hline
\end{tabular}

${ }^{\mathrm{z}}$ Allele sizes in brackets are the corresponding sizes of previously published alleles without the 18-bp M13 tail (Mejía et al., 2011). marker allele can be followed during introgression without hindrance from equal-sized Muscadinia alleles.

Segregation patterns of the p3_VvAGL11 marker followed expected 1:1:1:1 ratios for all progenies except Ga. 14-20, which had an unexplained excess of the 212/204 genotype (Table 1). Offtype plants nearly always had one of the three Muscadinia alleles, indicating that small amounts of windblown pollen may be entering the mesh bags used for pollination because NC74CO49-10 blooms at the same time as nearby muscadine vines.

NC74CO49-10 is a female complex hybrid between $V$. vinifera and $V$. rotundifolia (Fig. 1) with the reputation of being fertile with both progenitors, although we could find no published literature attesting to this fact. NC74CO49-10 was moderately fertile when crossed to Euvitis in this study, and we were able to produce fully developed seed without difficulty when crossing it to a several Euvitis cultivars. However, analysis of the hybrid progenies from NC74CO49-10 encountered the same problems as previous efforts at producing Euvitis $\times$ Muscadinia hybrids (Dunstan, 1962, 1963; Fry, 1964; Jelenković and Olmo, 1968, 1969; Patel and Olmo, 1955). Initial germination and seedling growth in the greenhouse were usually similar to pure Muscadinia crosses. However, once planted in the field, many hybrid vines suffered from poor growth and eventually died. Other vines had excellent initial growth only to dieback to the ground over the winter. Symptoms of Pierce's disease (Xylella fastidiosa Wells et al.) were common, and this disease likely accounted for significant vine dieback and death, especially in progenies derived from pure $V$. vinifera seedless parents. Because of low vigor and stem dieback, most vines that fruited did so in only 1 or 2 of the 3 years encompassed by this study. Hybrid seedlings ranged from fully fertile to quite sterile as judged by fruit-set, with many vines producing only a small number of berries. The production of small shot-sized berries was common and is likely a result of parthenocarpic fruit-set. Many vines produced a mixture of larger sized berries with seed and smaller berries less than one-third as large without seed or seed traces. Because it has been demonstrated that berry size increases with SN (Olmo, 1946), in these cases, only the larger seeded berries were examined as small berries are likely parthenocarpic and do not indicate the presence of stenospermocarpic seedlessness.

Stenospermocarpic seedlessness is a complex quantitative trait and seedless vines can vary from near complete seedlessness to producing very large seed traces, and seed size is influenced by genetic background (Mejía et al., 2011). Seedlessness in the hybrid seedlings was evaluated both quantitatively by measuring

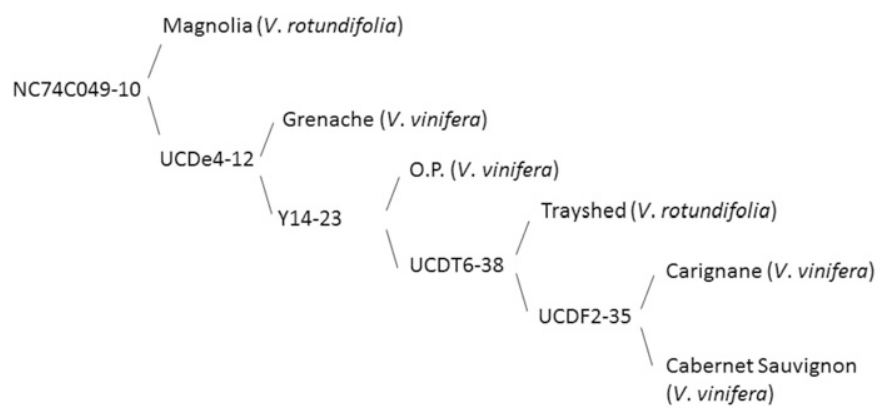

Fig. 1. Partial lineage of Euvitis $\times$ Muscadinia grape hybrid NC74C049-10 (Goldy et al., 1986). 
seed weight and qualitatively by assigning each seed to one of four classes. Seed qualitative classification was effective as measured by high correlation between MSC and quantitative measures of seed size (Table 3). All quantitative measures of seed development and BW were highly correlated, except for $\mathrm{MBW} \times \mathrm{BWCS}$.

Because of the difficulty in organoleptic evaluation of the stenospermocarpic trait, Ledbetter et al. (1994) advised using quantitative measures of seed development such as relative seed mass in determining seedlessness in progenies. There were significant differences between seedlings possessing the 214-bp p3_VvAGL11 allele and those that did not in all quantitative measures of seed development (Table 4). MSFW was almost 3 -fold lower in seedlings that displayed the 214-bp allele. The 24-mg MSFW average for seedlings with the 214-bp allele falls below the 25-mg limit used by Ramming et al. (1990) to define seedlessness. MSWB and BWCS were about half in seedlings that carry the 214-bp allele as those which did not have the allele. These differences strongly indicate that seedlings with the 214-bp allele have less seed development than those that do not have the 214-bp allele. MSC was 3.5 in those seedlings without the allele, indicating a high degree of normal seed development. Seed from vines with the 214-bp allele varied from class 0 to class 4 , with an average of 2.2 overall. MBW

Table 3. Spearman correlation coefficients between mean seed fresh weight (MSFW), mean seed weight per berry (MSWB), mean berry weight (MBW), percent berry weight composed of seed (BWCS), and mean seed classification (MSC) in Euvitis $\times$ Muscadinia grape hybrid seedlings. ${ }^{\mathrm{z}}$

\begin{tabular}{lcccc}
\hline & MSFW & MSWB & MBW & BWCS \\
\hline MSWB & $0.952^{*}$ & & & \\
MBW & $0.629^{*}$ & $0.606^{*}$ & & \\
BWCS $^{*}$ & $0.796^{*}$ & $0.853^{*}$ & NS & \\
MSC $^{\text {y }}$ & $0.853^{*}$ & $0.830^{*}$ & $0.532^{*}$ & $0.709^{*}$
\end{tabular}

${ }^{\mathrm{z}}$ Results were obtained using all fruiting plants from six hybrid progenies across multiple years.

${ }^{\mathrm{y}}$ The four seed classification categories used were 1) not evaluable aborted ovules or seeds, 2) rudimentary seeds, 3) complete but unlignified seeds, and 4) complete and lignified seeds.

*, ns Significant at $P<0.001$ and nonsignifcant.

Table 4. Effect of the 214-bp seedlessness-associated p3_VvAGL11 allele on mean seed fresh weight (MSFW), mean seed weight per berry (MSWB), mean berry weight (MBW), and percent berry weight composed of seed (BWCS), and mean seed classification (MSC) in Euvitis $\times$ Muscadinia grape hybrid seedlings.

\begin{tabular}{lccccc}
\hline Allele $^{\mathrm{z}}$ & MSFW (mg) & MSWB (mg) & MBW (g) & BWCS (\%) & MSC $^{\mathrm{y}}$ \\
\hline+214 & 19 & 24 & 1.14 & 2.0 & 2.2 \\
-214 & 49 & 53 & 1.51 & 3.6 & 3.5 \\
Sig. & $<0.001^{\mathrm{x}}$ & $<0.001^{\mathrm{x}}$ & $<0.001^{\mathrm{x}}$ & $<0.001^{\mathrm{x}}$ & $<0.001^{\mathrm{w}}$
\end{tabular}

${ }^{\mathrm{z}}$ Progeny with one copy of the 214-bp seedlessness-associated p3_VvAGL11 allele $(+214)$ vs. progeny without the 214-bp seedlessness-associated p3_VvAGL11 allele $(-214)$.

${ }^{\mathrm{y}}$ The four seed classification categories used were 1) not evaluable aborted ovules or seeds, 2) rudimentary seeds, 3) complete but unlignified seeds, and 4) complete and lignified seeds.

${ }^{\mathrm{x}}$ Significance determined by the Mann-Whitney rank sum test.

${ }^{\mathrm{w}}$ Significance determined by a $t$ test.

Results were obtained using all fruiting plants from six hybrid progenies across multiple years. was higher in vines that lacked the 214-bp allele. This may be due to pleiotropic effect through growth regulators produced by the developing seed that influence berry growth, one or more linked independent genes, or both having a direct effect on berry growth (Ledbetter and Ramming, 1989).

The phenotypic definition of stenospermocarpic seedlessness can vary with several factors involved including seed trace size, ovule abortion, hardness of the seed coat, the extent of endosperm development, and environmental conditions (Ledbetter and Ramming, 1989; Owens, 2008). For this study, the lack of a lignified seed coat (class 4 ) was used as the primary determinate of seedlessness, with the presence of any amount of lignified seed used as the definition of a vine being seeded. Using this qualitative standard, the p3_VvAGL11 marker was able to correctly predict the presence or absence of seedlessness in 79 of 93 vines (Table 5). Five vines were false negatives, i.e., the marker predicted them to be seeded when they did not in fact produce any lignified seed. If these are truly stenospermocarpic vines, then the use of the marker as a selection tool would result in useful genotypes being lost. In examining $371 \mathrm{~F}_{1} \mathrm{~V}$. vinifera hybrids, Bergamini et al. (2013) did not find any false negatives. However, the germplasm used in this study differs from $V$. vinifera hybrids in that there is a high degree of sterility and parthenocarpic fruit-set, and it is likely that most of these false negatives are not stenospermocarpic seedless but are rather seeded vines producing parthenocarpic fruit. In support of this theory, four of five of the false-negative seedlings produced very small berries less than $1 \mathrm{~g}$ in weight (data not shown), which is lower than the average of those containing the 214-bp allele.

Nine vines were false positives, in which the vine had the 214-bp allele but also produced lignified seed (Table 5). From a breeding standpoint, this error is not as troubling as a false negative becasue the vines will be discarded later when the fruit is evaluated. Although there is a small cost incurred for growing vines that are not useful, it is much less than if the marker had not been used. In their examination of $V$. vinifera germplasm, Bergamini et al. (2013) found a few instances of seeded cultivars with the seedless p3_VvAGL11 marker allele, resulting in a 1.9\% false-positive rate. This is less than the 9.7\% (9 of 93) falsepositive rate in our study (Table 5). However, qualitative classification of seedlessness is difficult (Ledbetter et al., 1994) and the low BN produced by many of these hybrid seedlings hampered our ability to reliably qualitatively score these seedlings. In addition, the differing genetic background of these hybrids may also result in less penetrance of the dominant seedless trait.

Despite the $9.7 \%$ false-positive rate and the $5.4 \%$ falsenegative rate, the p3_VvAGL11 marker will facilitate the introgression of the seedless trait into Muscadinia. Selection

Table 5. Effect of the 214-bp seedlessness-associated p3_VvAGL11 allele on the presence of lignified seed (seed classification 4) in Euvitis $\times$ Muscadinia grape hybrid progenies. ${ }^{\mathrm{z}}$

\begin{tabular}{lcrr}
\hline & \multicolumn{3}{c}{ Lignified seed } \\
\cline { 3 - 4 } & & Yes & No \\
\hline Allele $^{y}$ & +214 & 9 & 38 \\
& -214 & 41 & 5
\end{tabular}

${ }^{\mathrm{z}}$ Results were obtained using all fruiting plants from six hybrid progenies across multiple years.

${ }^{y}$ Progeny with one copy of the 214-bp seedlessness-associated p3_VvAGL11 allele $(+214)$ vs. progeny without the 214-bp seedlessness-associated p3_VvAGL11 allele $(-214)$. 
for the 214-bp allele in the greenhouse will allow most seeded vines to be removed from progenies. More effort can then be expended on growing seedless vines. In addition, by removing most seeded vines, pollen can be more efficiently collected from vines the first year they bloom for immediate crossing or to save for future use. This is important because a large percentage of Euvitis $\times$ Muscadinia hybrid vines lack vigor and bloom only sporadically or only in a single year (Derman, 1964; Dunstan, 1963; Patel and Olmo, 1955). Selection with the p3_VvAGL11 marker will help to ensure that less time is wasted collecting pollen from seeded vines. The use of pollen from p3_VvAGL11-genotyped seedless vines immediately, before phenotyping, will allow early generations of introgression to proceed more quickly. The p3_VvAGL11 marker is also useful in discriminating between seeded vines with parthenocarpic fruit-set and stenospermocarpic vines. Finally, the p3_VvAGL11 marker may also be useful for introgressing traits other than seedlessness. Because Muscadinia and Euvitis do not share many alleles, the p3_VvAGL11 marker may be used to confirm hybridity at the seedling stage in crosses between the two subgenera.

\section{Literature Cited}

Basiouny, F.M. and D.G. Himelrick. 2001. Muscadine grapes. ASHS Press, Alexandria, VA.

Bergamini, C., M. Cardone, A. Anaclerio, R. Perniola, A. Pichierri, R. Genghi, V. Alba, L. Forleo, A. Caputo, C. Montemurro, A. Blanco, and D. Antonacci. 2013. Validation assay of p3_VvAGL11 marker in a wide range of genetic background for early selection of stenospermocarpy in Vitis vinifera L. Mol. Biotechnol. 54:1021-1030.

Bloodworth, J. 2014. SSC induction in Vitis muscadinia. U.S. Patent 9706726B2. Filed 15 Mar. 2013. Issued 18 July 2017.

Bouquet, A. and Y. Danglot. 1996. Inheritance of seedlessness in grapevine (Vitis vinifera L.). Vitis 35:35-42.

Clark, J. and J. Moore. 1999. 'Jupiter' seedless grape. HortScience 34:1297-1299.

Conner, P., J. Conner, P. Catotti, J. Lewter, J. Clark, and L. Biasi. 2017. Development and characterization of molecular markers associated with female plants in muscadine grape. J. Amer. Soc. Hort. Sci. 142:143-150.

Derman, H. 1964. Cytogenetics in hybridization of bunch- and muscadine-type grapes. Econ. Bot. 18:137-148.

Dunstan, R. 1962. Some fertile hybrids of bunch and muscadine grapes. J. Hered. 53:299-303.

Dunstan, R. 1963. Hybridization of Euvitis $\times$ Vitis rotundifolia: Backcrosses to muscadine. Proc. Amer. Soc. Hort. Sci. 84:238-242.

Fry, B. 1964. Fertile interspecific hybrids Vitis rotundifolia $\times$ Vitis vinifera. Georgia Agr. Expt. Sta. Mimeograph Ser. N.S. 200.

Goldy, R., W. Ballinger, and E. Maness. 1986. Fruit anthocyanin content of some Euvitis $\times V$. Rotundifolia hybrids. J. Amer. Soc. Hort. Sci. 111:955-960.
Grimplet, J., A. Adam-Blondon, P. Bert, O. Bitz, D. Cantu, C. Davies, S. Delrot, M. Pezzotti, S. Rombauts, and G. Cramer. 2014. The grapevine gene nomenclature system. BMC Genomics 15:1077.

Jelenković, G. and H. Olmo. 1968. Cytogenetics of Vitis: III. Partially fertile $\mathrm{F}_{1}$ diploid hybrids between $V$. vinifera $\times$ Vitis rotundifolia Michx. Vitis 7:281-293.

Jelenković, G. and H. Olmo. 1969. Cytogenetics of Vitis: IV. Backcross derivatives of $V$. vinifera $\times$ Vitis rotundifolia Michx. Vitis 8:1-11.

Karaagac, E., A. Vargas, M. de Andrés, I. Carreño, J. Ibáñez, J. Carreño, J. Martínez-Zapater, and J. Cabezas. 2012. Marker assisted selection for seedlessness in table grape breeding. Tree Genet. Genomes 8:1003-1015.

Lane, R. 1997. Breeding muscadine and southern bunch grapes. Fruit Var. J. 51:144-148.

Ledbetter, C., L. Burgos, and D. Palmquist. 1994. Comparison of methods for determining the stenospermocarpic trait in Vitis vinifera L. Vitis 33:11-13.

Ledbetter, C. and D. Ramming. 1989. Seedlessness in grapes. Hort. Rev. 11:159-184.

Lu, J., L. Schell, and S. Lamikanra. 1993. Introgression of seedlessness from bunch grapes into muscadine grapes. Proc. Florida State Hort. Soc. 106:122-124.

Malabarba, J., V. Buffon, J. Mariath, M. Gaeta, M. Dornelas, M. Margis-Pinheiro, and L. Revers. 2017. The MADS-box gene agamous-like 11 is essential for seed morphogenesis in grapevine. J. Expt. Bot. 68:1493-1506.

Mejía, N., B. Soto, M. Guerrero, X. Casanueva, C. Houel, M. Miccono, R. Ramos, L. Le Cunff, J. Boursiquot, P. Hinrichsen, and A. AdamBlondon. 2011. Molecular, genetic and transcriptional evidence for a role of $V v A G L 11$ in stenospermocarpic seedlessness in grapevine. BMC Plant Biol. 11:57.

Olmo, H. 1946. Correlations between seed and berry development in some seeded varieties of Vitis vinifera. Proc. Amer. Soc. Hort. Sci. 48:291-297.

Owens, C. 2008. Grapes, p. 197-233. In: J. Hancock (ed.). Temperate fruit crop breeding. Springer Science+Business Media, Berlin, Germany.

Patel, G. and H. Olmo. 1955. Cytogenetics of Vitis: I. The hybrid V. vinifera $\times$ Vitis rotundifolia. Amer. J. Bot. 42:141-159.

Ramming, D., C. Ledbetter, and R. Tarailo. 1990. Hybridization of seedless grapes. Vitis 29:439-444.

Reisch, B., D. Peterson, R. Pool, and M. Martens. 1993. Table grape varieties for cool climates. Cornell Info. Bul. 234

Rounsley, S., G. Ditta, and M. Yanofsky. 1995. Diverse roles for MADS box genes in Arabidopsis development. Plant Cell 7:12591269.

Striem, M., G. Ben-Hayyim, and P. Spiegel-Roy. 1996. Identifying molecular genetic markers associated with seedlessness in grape. J. Amer. Soc. Hort. Sci. 121:758-763.

Sutton, J., B. Robertson, and I. Jamieson. 2011. Dye shift: A neglected source of genotyping error in molecular ecology. Mol. Ecol. Resources 11:514-520. 
Supplemental Table 1. Genotype of the p3_VvAGL11 sequence-tagged site marker in Euvitis and Muscadinia grape germplasm.

\begin{tabular}{|c|c|c|c|c|}
\hline Cultivar & Subgenus & Seedlessness trait & p3_VvAGL11 allele 1 & p3_VvAGL11 allele 2 \\
\hline Blue Lake & Euvitis & Seeded & 192 & 194 \\
\hline Cabernet & Euvitis & Seeded & 204 & 212 \\
\hline Caco & Euvitis & Seeded & 194 & 204 \\
\hline Concord & Euvitis & Seeded & 194 & 204 \\
\hline Conquistador & Euvitis & Seeded & 194 & 204 \\
\hline Golden Muscat & Euvitis & Seeded & 194 & 204 \\
\hline Kissamee & Euvitis & Seeded & 192 & 194 \\
\hline Lady Finger & Euvitis & Seeded & 204 & 204 \\
\hline Malbec & Euvitis & Seeded & 204 & 204 \\
\hline Merlot & Euvitis & Seeded & 204 & 204 \\
\hline Miss Blanc & Euvitis & Seeded & 192 & 200 \\
\hline Pinot Noir & Euvitis & Seeded & 204 & 204 \\
\hline Victoria Red & Euvitis & Seeded & 208 & 208 \\
\hline Zinfandel & Euvitis & Seeded & 204 & 204 \\
\hline$A-3006^{z}$ & Euvitis & Seedless & 204 & 214 \\
\hline Autumn Royal & Euvitis & Seedless & 208 & 214 \\
\hline Black Monukka & Euvitis & Seedless & 208 & 214 \\
\hline C49-96 & Euvitis & Seedless & 204 & 214 \\
\hline Concord Seedless & Euvitis & Seedless & Null $^{\mathrm{x}}$ & 214 \\
\hline Crimson Seedless & Euvitis & Seedless & 204 & 214 \\
\hline Faith & Euvitis & Seedless & 200 & 214 \\
\hline Flame Seedless & Euvitis & Seedless & 204 & 214 \\
\hline Joy & Euvitis & Seedless & 200 & 214 \\
\hline Y139-139-04 & Euvitis & Seedless & 204 & 214 \\
\hline Y539-144-06 & Euvitis & Seedless & 204 & 214 \\
\hline Alachua & Muscadinia & Seeded & 196 & 196 \\
\hline Albermarle & Muscadinia & Seeded & 194 & 196 \\
\hline Black Beauty & Muscadinia & Seeded & 196 & 196 \\
\hline Bountiful & Muscadinia & Seeded & 194 & 196 \\
\hline Burgaw & Muscadinia & Seeded & 194 & 200 \\
\hline Carlos & Muscadinia & Seeded & 196 & 200 \\
\hline Chowan & Muscadinia & Seeded & 196 & 196 \\
\hline Cowart & Muscadinia & Seeded & 196 & 196 \\
\hline Darlene & Muscadinia & Seeded & 196 & 196 \\
\hline Dawn & Muscadinia & Seeded & 196 & 196 \\
\hline Dearing & Muscadinia & Seeded & 194 & 196 \\
\hline Dixie & Muscadinia & Seeded & 194 & 200 \\
\hline Dixieland & Muscadinia & Seeded & 196 & 196 \\
\hline Eudora & Muscadinia & Seeded & 196 & 196 \\
\hline Fennell's 3-way Hybrid & Muscadinia & Seeded & 194 & 194 \\
\hline Flowers & Muscadinia & Seeded & 194 & 196 \\
\hline Fry & Muscadinia & Seeded & 196 & 196 \\
\hline Ga. $10-1-294^{w}$ & Muscadinia & Seeded & 196 & 196 \\
\hline Ga. $11-19^{w}$ & Muscadinia & Seeded & 196 & 200 \\
\hline Ga. $1-2-112^{w}$ & Muscadinia & Seeded & 196 & 196 \\
\hline
\end{tabular}


Supplemental Table 1. Continued.

\begin{tabular}{|c|c|c|c|c|}
\hline Cultivar & Subgenus & Seedlessness trait & p3_VvAGL11 allele 1 & p3_VvAGL11 allele 2 \\
\hline Ga. $1-3-35^{w}$ & Muscadinia & Seeded & 196 & 200 \\
\hline Ga. $1-3-58^{w}$ & Muscadinia & Seeded & 200 & 200 \\
\hline Ga. $15-19-2^{w}$ & Muscadinia & Seeded & 196 & 196 \\
\hline Ga. $17-62^{w}$ & Muscadinia & Seeded & 196 & 196 \\
\hline Ga. $18-5^{\mathrm{w}}$ & Muscadinia & Seeded & 196 & 196 \\
\hline Ga. $2-8-10^{\mathrm{w}}$ & Muscadinia & Seeded & 196 & 196 \\
\hline Ga. $2-8-21^{\mathrm{w}}$ & Muscadinia & Seeded & 196 & 196 \\
\hline Ga. $4-3-147^{w}$ & Muscadinia & Seeded & 196 & 196 \\
\hline Ga. $4-4-2^{w}$ & Muscadinia & Seeded & 196 & 196 \\
\hline Ga. $5-1-34^{\mathrm{w}}$ & Muscadinia & Seeded & 196 & 196 \\
\hline Ga. $5-1-5^{\mathrm{w}}$ & Muscadinia & Seeded & 196 & 196 \\
\hline Ga. $6-2-187^{w}$ & Muscadinia & Seeded & 196 & 196 \\
\hline Ga. $6-2-192^{w}$ & Muscadinia & Seeded & 196 & 196 \\
\hline Ga. $6-2-26^{\mathrm{w}}$ & Muscadinia & Seeded & 196 & 196 \\
\hline Ga. $6-2-46^{\mathrm{w}}$ & Muscadinia & Seeded & 196 & 196 \\
\hline Ga. $6-2-64^{\mathrm{w}}$ & Muscadinia & Seeded & 196 & 196 \\
\hline Ga. $6-2-78^{w}$ & Muscadinia & Seeded & 196 & 196 \\
\hline Ga. $6-5-2^{w}$ & Muscadinia & Seeded & 196 & 196 \\
\hline Ga. $6-5-5^{\mathrm{w}}$ & Muscadinia & Seeded & 196 & 196 \\
\hline Ga. $6-6-358^{w}$ & Muscadinia & Seeded & 196 & 196 \\
\hline Ga. $6-9-102^{w}$ & Muscadinia & Seeded & 196 & 196 \\
\hline Ga. $8-1-338^{w}$ & Muscadinia & Seeded & 196 & 196 \\
\hline Ga. $8-1-54^{\mathrm{w}}$ & Muscadinia & Seeded & 196 & 196 \\
\hline Hall & Muscadinia & Seeded & 196 & 196 \\
\hline Higgins & Muscadinia & Seeded & 196 & 196 \\
\hline Hunt & Muscadinia & Seeded & 196 & 196 \\
\hline Ison & Muscadinia & Seeded & 196 & 196 \\
\hline James & Muscadinia & Seeded & 194 & 196 \\
\hline Janet & Muscadinia & Seeded & 196 & 200 \\
\hline Jumbo & Muscadinia & Seeded & 194 & 196 \\
\hline La Salle & Muscadinia & Seeded & 194 & 196 \\
\hline Lake Charles & Muscadinia & Seeded & 196 & 196 \\
\hline Lane & Muscadinia & Seeded & 196 & 196 \\
\hline Late Fry & Muscadinia & Seeded & 196 & 196 \\
\hline Latham & Muscadinia & Seeded & 196 & 196 \\
\hline Magnolia & Muscadinia & Seeded & 196 & 200 \\
\hline Magoon & Muscadinia & Seeded & 196 & 196 \\
\hline Marsh & Muscadinia & Seeded & 194 & 194 \\
\hline Memory & Muscadinia & Seeded & 196 & 196 \\
\hline Nesbitt & Muscadinia & Seeded & 196 & 196 \\
\hline Noble & Muscadinia & Seeded & 196 & 200 \\
\hline Pamlico & Muscadinia & Seeded & 194 & 196 \\
\hline Pineapple & Muscadinia & Seeded & 196 & 200 \\
\hline Polyanna & Muscadinia & Seeded & 196 & 196 \\
\hline Pride & Muscadinia & Seeded & 196 & 196 \\
\hline
\end{tabular}


Supplemental Table 1. Continued.

\begin{tabular}{|c|c|c|c|c|}
\hline Cultivar & Subgenus & Seedlessness trait & p3_VvAGL11 allele 1 & p3_VvAGL11 allele 2 \\
\hline Regale & Muscadinia & Seeded & 194 & 196 \\
\hline Roanoke & Muscadinia & Seeded & 194 & 200 \\
\hline Rosa & Muscadinia & Seeded & 194 & 196 \\
\hline San Jacinto & Muscadinia & Seeded & 194 & 196 \\
\hline Scarlett & Muscadinia & Seeded & 196 & 196 \\
\hline Scuppernong & Muscadinia & Seeded & 194 & 196 \\
\hline Sterling & Muscadinia & Seeded & 194 & 194 \\
\hline Sugar Gate & Muscadinia & Seeded & 196 & 196 \\
\hline Summit & Muscadinia & Seeded & 196 & 196 \\
\hline Supreme & Muscadinia & Seeded & 196 & 196 \\
\hline Sweet Jenny & Muscadinia & Seeded & 196 & 196 \\
\hline Tara & Muscadinia & Seeded & 196 & 196 \\
\hline Top Sail & Muscadinia & Seeded & 194 & 196 \\
\hline Triumph & Muscadinia & Seeded & 196 & 196 \\
\hline$V$. popenoi & Muscadinia & Seeded & 194 & 194 \\
\hline Welder & Muscadinia & Seeded & 196 & 200 \\
\hline
\end{tabular}

${ }^{\mathrm{z}}$ Seedless Euvitis selection from the University of Arkansas grape breeding program.

${ }^{\mathrm{y}}$ Seedless Euvitis selection from the U.S. Department of Agriculture table grape breeding program, courtesy of David Ramming.

${ }^{\mathrm{x}}$ Presence of null allele was determined by the production of a single peak at $214 \mathrm{bp}$ and progeny ratios consistent with a 1:1:1:1 segregation pattern.

${ }^{\mathrm{w}}$ Selection from the University of Georgia muscadine breeding program. 